Trauma Berufskrankh $2003 \cdot 5$ [Suppl 1]: S67-S72 DOI 10.1007/s10039-002-0661-4

Fehlheilungen

Klaus Seide · Christian Jürgens · Michael Wenzl · Stefan Fuchs

BG-Unfallkrankenhaus Hamburg

\title{
Achskorrekturen und Pseudarthrosenbehandlung nach Unterschenkelfrakturen durch minimalinvasiven Zugang und Fixateur interne
}

\section{Zusammenfassung}

Ein wichtiges Prinzip der Behandlung von Pseudarthrosen ist die schonende operative Korrektur mit minimaler Weichteilschädigung, um eine eingeschränkte Blutversorgung nicht zusätzlich zu beeinträchtigen. 8 Umstellungsosteotomien sowie 36 Pseudarthrosenbehandlungen am Unterschenkel wurden mit einem minimalinvasiv implantierbaren multidirektionalen Fixateur interne, 1 Behandlung mit einem neu entwickelten unaufgebohrten Marknagel, der nach dem gleichen Prinzip winkelstabil verriegelt werden kann, durchgeführt. In allen Fällen kam es zur knöchernen Konsolidierung. An Komplikationen wurden 2 Plattenlagerinfekte und 10 steitis beobachtet. In allen diesen Fällen handelte es sich um Patienten mit einer primären Infektpseudarthrose. In 1 Fall erfolgte eine unzureichende Korrektur mit einer verbliebenen Fehlstellung von $10^{\circ} \mathrm{Au}$ ßenrotation. Die Kombinationen eines winkelstabilen Implantats mit einem minimalinvasiven Zugang und der winkelstabile Verriegelungsnagel stellen $u$. E. gute Methoden für die Behandlung von Fehlheilungen am Unterschenkel dar.

\section{Schlüsselwörter}

Pseudarthrose $\cdot$ Fehlstellung $\cdot$ Tibia $\cdot$ Fixateur interne $\cdot$ Nagel $\cdot$ Winkelstabil
A frund der problematischen Durchblutungssituation der Tibia sind insbesondere nach Mehrfragmentfrakturen in bis zu etwa $16 \%$ der Fälle Pseudarthrosen zu beobachten $[2,3,6]$. Auch nach offenen Frakturen, die primär mit dem Fixateur externe behandelt wurden, besteht eine hohe Inzidenz von Pseudarthrosen. Gleichermaßen finden sich bei dieser Art der Versorgung nicht selten Achsfehler. Achsfehlstellungen sind auch nach Marknagelosteosynthesen bei Frakturen im weit proximalen oder weit distalen Anteil der Tibia zu beobachten.

Ein wichtiges Prinzip der Behandlung dieser Fehlheilungen ist eine möglichst schonende operative Korrektur mit minimaler Frakturfreilegung, um die ohnehin schon eingeschränkte Blutversorgung nicht zusätzlich zu beeinträchtigen. Neben verschiedenen Verfahren der Nagelung (aufgebohrt, Kompression $[8,9,11,12])$ und des Fixateur externe [5] sowie der DC-Plattenosteosynthese stehen mit dem Fixateur interne [14] und dem winkelstabilen Nagel 2 weitere Systeme zur Verfügung. Über Indikationen und Ergebnisse bei diesen Verfahren wird im Folgenden berichtet.

\section{Material und Methode}

Zwischen September 1997 und September 2000 wurden 36 Pseudarthrosenbehandlungen und 8 Umstellungsosteotomien am Unterschenkel mit dem Fixateur interne durchgeführt. Alle
Patienten wurden nach Abschluss der knöchernen Konsolidierung nachuntersucht.

Die verwendeten Titanplattenfixateure (TiFix, Fa. Litos, Hamburg) beruhen auf dem Prinzip der multidirektionalen Winkelstabilität [13] (Abb. 1). Der Schraubenkopf wird mit einem Gewinde fest in der Platte verankert. Ein besonderer Vorteil ist, dass durch die Verwendung verschiedener Titanhärten eine Gewindeformung im Schraubenloch der Platte auch bis zu $20^{\circ}$ von der Senkrechten in allen Richtungen abweichend erfolgen kann. Von dem System stehen 2 Varianten zur Auswahl, bei der ersten wird das Gewinde in der Platte mit einem Schraubendränger vorgedrängt, bei der zweiten ist das Schraubenloch zu einer Lippe verjüngt, welche sich beim Eindrehen der Schraube in der entsprechenden Richtung umformt. Versuche haben gezeigt, dass es dabei durch Aufbrechen der sich auf jeder Titanfläche bildenden harten Titanoxidschicht zu atomaren Reaktionen mit einer Kaltverschweißung an einigen Punkten des Gewindes kommt. Hierdurch ergibt sich

๑) Springer-Verlag 2003

Priv.-Doz.Dr. Klaus Seide BG-Unfallkrankenhaus Hamburg, Bergedorfer Straße 10,21033 Hamburg E-Mail: k.seide@buk-hamburg.de, Phone: 040-73062701, Fax: 040-73062703 
Trauma Berufskrankh $2003 \cdot 5$ [Suppl 1]: S67-S72 DOI 10.1007/s10039-002-0661-4

\section{K. Seide $\cdot$ C. Jürgens $\cdot$ M. Wenzl $\cdot$ S. Fuchs}

\section{Deformity corrections and treatment of nonunions} by a minimally invasive approach and application of an internal fixator after crural fractures

\section{Abstract}

One of the basic principles in the treatment of nonunion and malunion is gentle operative correction with minimal damage to the soft tissues, to avoid any further impairment of the already restricted blood flow. Eight tibial correction osteotomies and 36 stabilisations of tibial nonunion were performed by application of an internal fixator (locked screw-plate interface) that can be implanted by way of a minimally invasive approach; in one case an intramedullary nail with stable-angle locking screws was applied. Consolidation was achieved in all cases. Two cases of plate infection and one of osteitis were observed, all in patients who had had a primary infection associated with nonunion. In one case a residual deformity of $10^{\circ}$ torsion remained. In our judgement, the combination of an internal fixator with stable-angle locking screws and a minimally invasive approach and also the stable-angle locking nail are successful methods of treatment for tibial malunion or nonunion.

\section{Keywords}

Pseudarthrosis - Malunion · Tibia $\cdot$ Internal fixator $\cdot$ Nail $\cdot$ Angular stability $\cdot$ Locked screw-plate interface

\section{Fehlheilungen}

eine hohe Festigkeit, die Verbindungen sind jedoch im Rahmen der Metallentfernung lösbar [14].

In bisher 1 Fall wurde ein neu entwickelter unaufgebohrter Marknagel, der im Verriegelungsloch nach dem oben beschriebenen Prinzip eine Lippe aufweist und mit gewindetragenden Verriegelungsbolzen verriegelt werden kann, verwendet. Vorversuche haben gezeigt, dass es bei diesem winkelstabil verriegelten Nagel zu einer deutlichen Stabilitätserhöhung, insbesondere der Torsion, kommt [13].

\section{Ergebnisse}

Bei den Pseudarthrosen handelte es sich um

- 3 proximale,

- 20 diaphysäre und

- 13 distale Pseudarthrosen.

Vorausgegangene Osteosynthesen ( Tabelle 1) waren

- in 17 Fällen Fixateur-externe-Montagen,

- in 11 Fällen Marknagelosteosynthesen,

- in 7 Fällen Plattenosteosynthesen sowie

- in 1 Fall eine Klammer-Versorgung.

Im Mittel waren 1,9 (1-4) Operationen vorausgegangen.

In allen Fällen kam es zur knöchernen Konsolidierung. Die Heilungsdauer betrug im Mittel 15 Wochen (Minimum 6 Wochen, Maximum 26 Wochen) (Abb. 2, 3). Die Kriterien einer verzögerten Heilung ergaben sich dabei in 2 Fällen. In 1 Fall wurde eine Spongiosaplastik erforderlich.
An Komplikationen wurden 2 Plattenlagerinfekte und 1 Osteitis beobachtet. In allen diesen Fällen handelte es sich um Patienten mit einer primären Infektpseudarthrose. In 1 Fall erfolgte eine unzureichende Korrektur mit einer verbliebenen Fehlstellung von $10^{\circ} \mathrm{Au}-$ Benrotation bei erfolgter Achskorrektur. Darüber hinaus war eine Unterschenkelvenenthrombose zu verzeichnen.

Der von uns neu entwickelte winkelstabile Verriegelungsnagel wurde bisher in 1 Fall eingesetzt. Der Operationsverlauf und die Handhabung des Systems zeigten keine wesentlichen Unterschiede zur Vorgehensweise mit einem herkömmlichen unaufgebohrten Tibianagel. Lediglich das Bohren der Löcher für die Verriegelungsschrauben in 2 Durchmessern stellte eine leichte Modifikation dar. Intraoperativ wurde der Eindruck einer stabilen Osteosynthese bestätigt. Nach 15 Wochen war die Fraktur röntgenologisch verheilt, der Patient belastete beschwerdefrei voll (Abb. 4).

\section{Diskussion}

Vorteile der winkelstabilen Plattensysteme (Fixateur-interne-Systeme) sind die zusätzliche Formstabilität und die dauerhaft flächenhafte Krafteinleitung auch bei überbrückenden Platten mit relativ wenig Schrauben. Direkter Knochenkontakt ist bei diesen Fixateursystemen ebenfalls nicht zwingend erforderlich, da die Krafteinleitung nicht wie bei herkömmlichen Plattensystemen durch Reibung im Bereich des Knochenplatteninterfaces, sondern direkt über die Schrauben-Platten-Verbindung erfolgt [10].

Aufgrund der hohen Stabilität kann daher auch die Anzahl der zur Stabili-
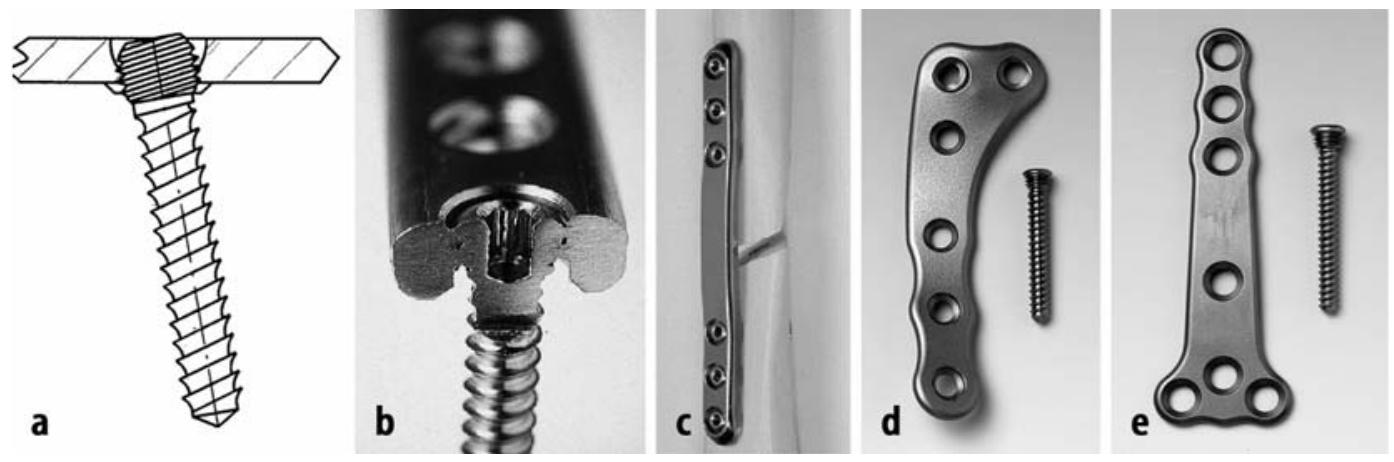

Abb. 1a-e $<$ Titanplattenfixateursystem (TiFix) mit der Möglichkeit einer multidirektionalen Schraubenlage: gedrängtes Gewinde oder Lippe im Plattenloch („weiches“ Titan Grad 0) und Gewinde im Schraubenkopf („,hartes“ Titan Grad 4) (a,b). Platten für den Unterschenkelschaft (c), den Tibiakopf (d) und das Pilon (e) 
Tabelle 1

Vorausgegangene Osteosynthesen bei 36 Pseudarthrosen

Lokalisation

Vorausgegangene Osteosynthesen

\begin{tabular}{llll}
\hline $\begin{array}{l}\text { Proximal } \\
\text { Diaphysär }\end{array}$ & $\begin{array}{l}1 \text { Klammer } \\
12 \text { Fixateur externe }\end{array}$ & 8 Nägel & \\
Distal & 5 Fixateur externe & 3 Nägel & 5 Platten
\end{tabular}

sierung erforderlichen Schrauben an beiden Seiten des Knochendefekts eingeschränkt werden. Daher lassen sich diese Fixateursysteme minimalinvasiv über kleine Inzisionen $[4,14]$ einbringen und am Knochen fixieren. In früherer Zeit wurden Pseudarthrosen und Fehlstellungen mit Plattenosteosynthesen, insbesondere unter Anwendung der dynamischen Kompression, in der Regel gut korrigiert und zur knöchernen Konsolidierung gebracht. Aufgrund der großflächigen Freilegung und einer relativ großen Masse an Osteosynthesematerial war der Eingriff mit einer Störung der lokalen Durchblutung verbunden.

Unter Verwendung eines Fixateur interne lassen sich die beiden wesentlichen Nachteile der Plattenosteosynthese vermeiden. Bei entsprechend kleiner Schnittführung ist die Schädigung des Weichteilmantels mit der eines Nagels, welcher bei der Indikationsstellung nicht selten konkurrierend in Erwägung zu ziehen ist, zu vergleichen. Gegenüber der Nagelung entfällt jedoch das Operationstrauma des Markraums. Dieses ist bei beruhigten Infektionen oder bei vorangegangenen Fixateur-externe-Osteosynthesen von besonderer Bedeutung, da in diesen Fällen ein deutlich erhöhtes Risiko einer Osteitis als Folge des Revisionseingriffs besteht. In einem solchen Fall ist eine nagelbedingte Markraumphlegmone weitaus schwerwiegender für den Patienten als begrenzte Infektionen im Bereich der Plattenschrauben oder des Plattenlagers.

Eine Weiterentwicklung des intramedullären Systems durch winkelstabil verriegelte Schrauben erscheint u. E. ebenfalls viel versprechend. Derzeit wird für die sichere Stabilisierung einer Pseudarthrose am Unterschenkel generell ein Aufbohren vorgeschlagen, um die erforderliche Stabilität im Pseudarthrosen- bereich zu erreichen $[8,11,12]$. Experimentelle Untersuchungen und der dargestellte klinische Fall bestätigen, dass durch die Winkelstabilität das Aufbohren wahrscheinlich entbehrlich ist. Den gleichen Zweck erfüllt die ebenfalls zur sicheren Stabilisierung führende Anwendung des Kompressionsmarknagels [9].

Die erfolgreiche Behandlung von therapierefraktären Pseudarthrosen erhöht die Lebensqualität der Patienten und ist von erheblicher ökonomischer Bedeutung [1]. Nach unseren Erfahrungen ist die Kombination eines winkelstabilen Implantats mit einem minimalinvasiven Zugang ein erfolgreiches Verfahren [7] und stellt zusammen mit dem vorgestellten winkelstabilen Verriegelungsnagel eine gute Ergänzung des Spektrums bisheriger Osteosynthesemethoden für die Behandlung von Pseudarthrosen am Unterschenkel dar.
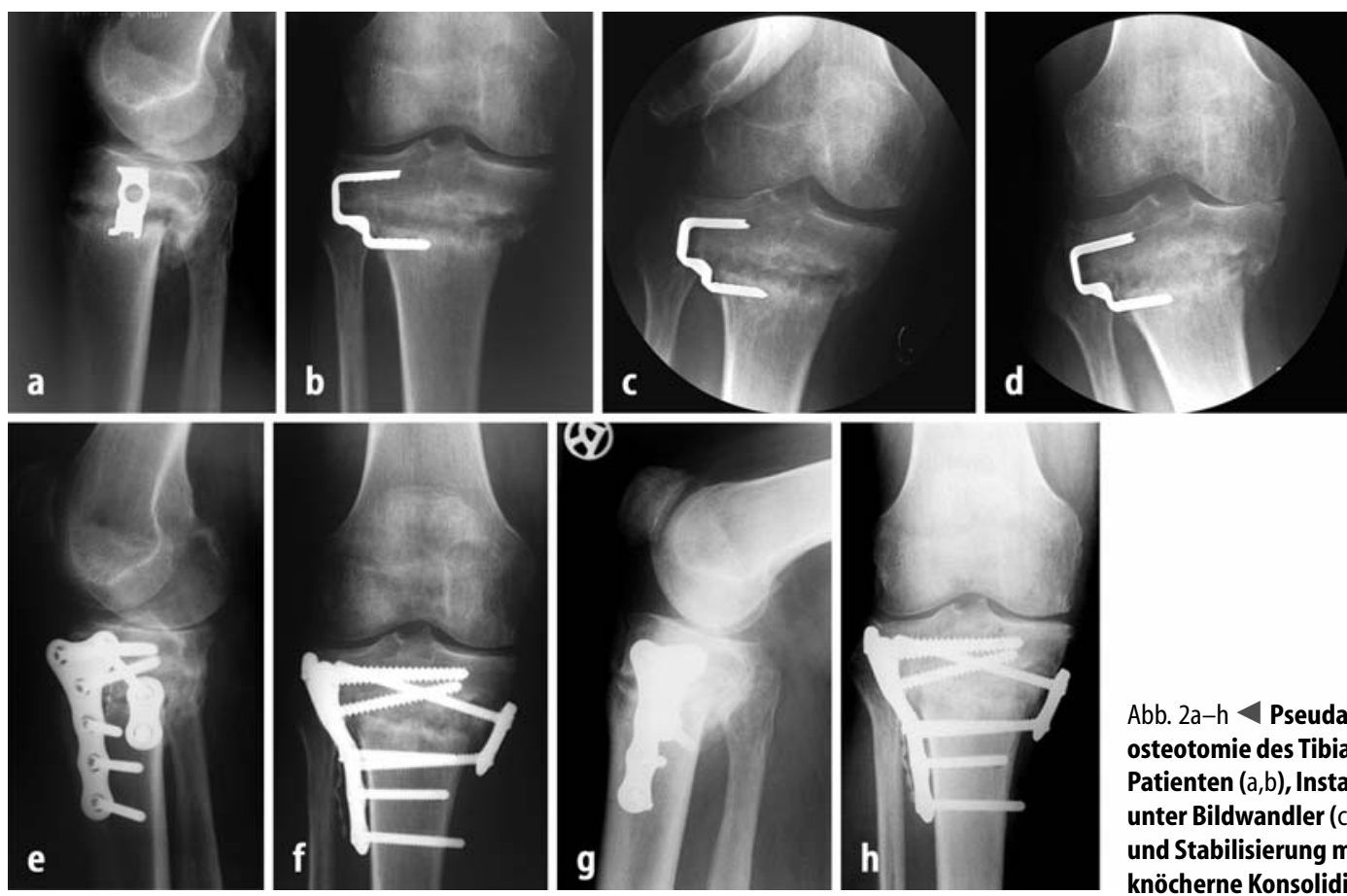

Abb. 2a- $\mathrm{h}<$ Pseudarthrose nach Umstellungsosteotomie des Tibiakopfs bei einem 54-jährigen Patienten (a,b), Instabilität der Pseudarthrose unter Bildwandler $(c, d)$, Pseudarthrosenrevision und Stabilisierung mit Fixateurs internes $(e, f)$, knöcherne Konsolidierung nach 3 Monaten $(g, h)$ 


\section{Fehlheilungen}
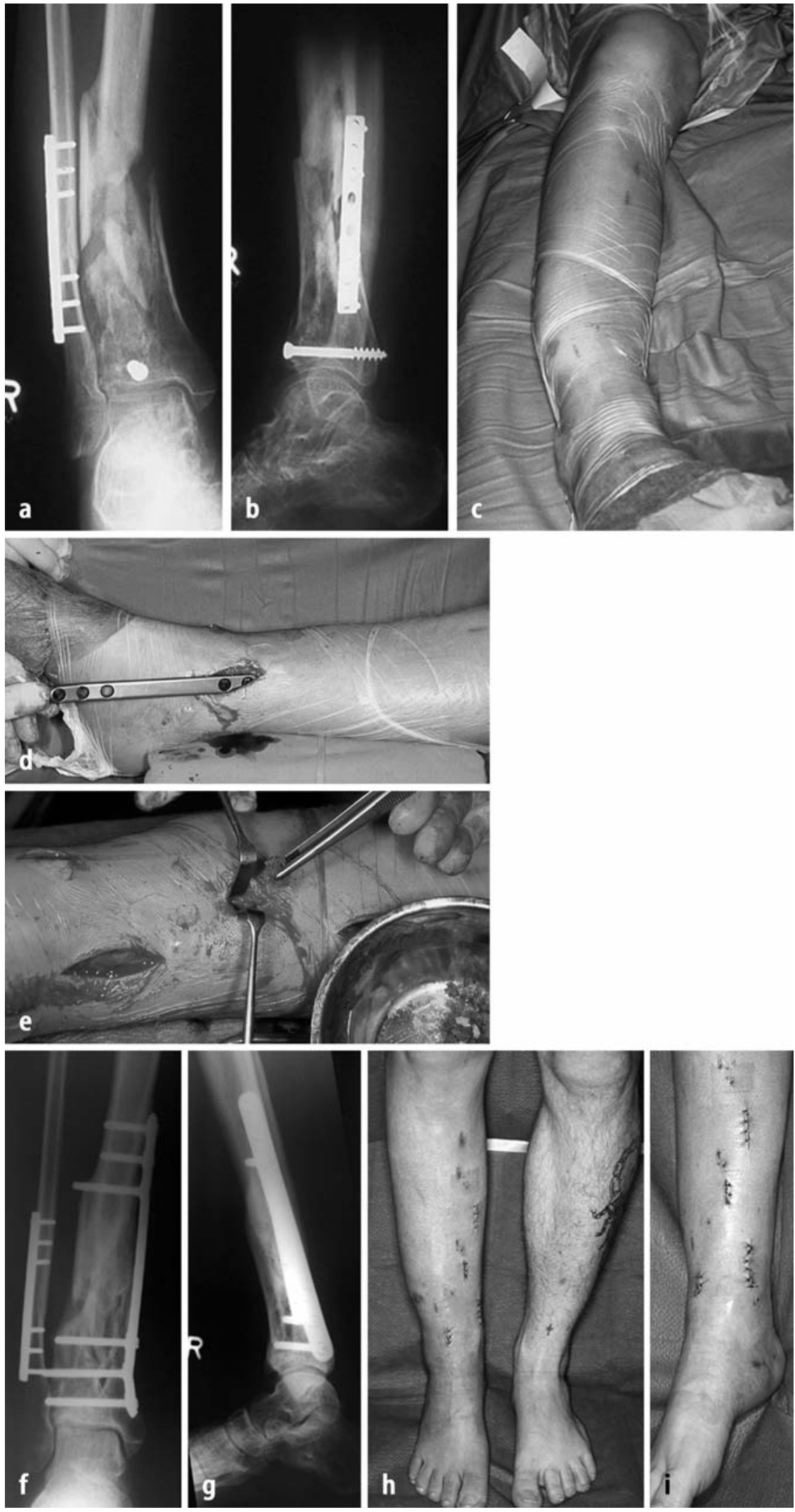


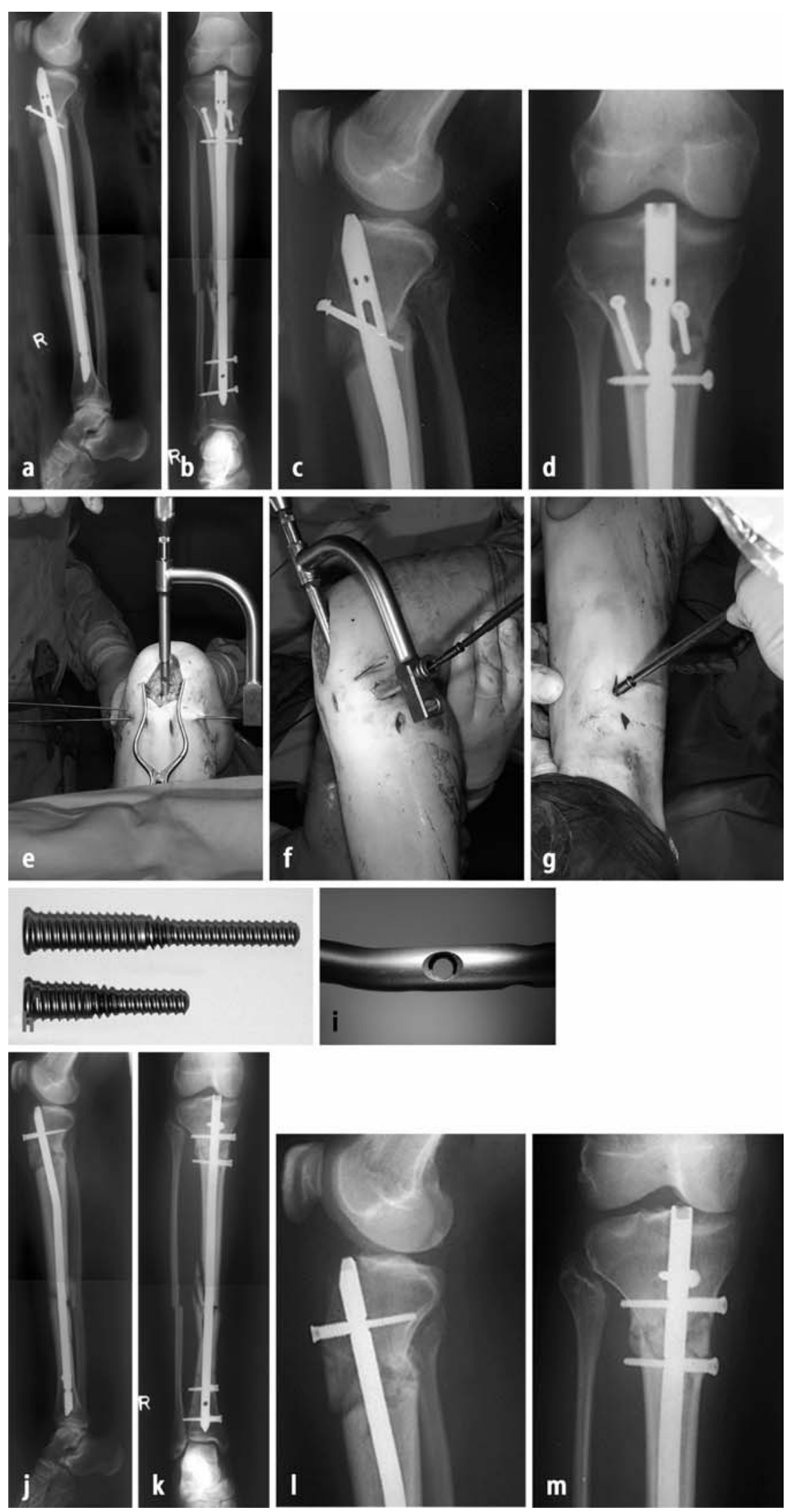

Abb. 4a-m 4 Fehlstellung nach 2-Etagen-Unterschenkelfraktur bei einer 24-jährigen Patientin. Marknagelung (UTN) und Zugschraubenosteosynthese 4 Monate zuvor (a-d), Wechsel des UTN gegen den winkelstabilen TiFix-Nagel mit Korrektur der Antekurvationsfehlstellung (e-g), Verriegelungsschrauben mit abgestuftem Gewinde und Schraubenloch mit Lippe $(h, i)$, radiologisches Ergebnis (j-m) 


\section{Fehlheilungen}

\section{Literatur}

1. Beaver R, Brinker MR, Barrack RL (1997) An analysis of the actual cost of tibial nonunions. J La State Med Soc 149: 200-206

2. Gaebler C, Berger U, Schandelmaier P, Greitbauer M, Schauwecker HH, Applegate B, Zych $G$, Vecsei V (2001) Rates and odds ratios for complications in closed and open tibial fractures treated with unreamed, small diameter tibial nails: a multicenter analysis of 467 cases. J Orthop Trauma 15: 415-423

3. Goldhahn S, Moser R, Bigler R, Matter P (2000) Behandlungsmethoden und -ergebnisse von Tibiaschaftfrakturen in der Schweiz. Eine prospektive multizentrische Studie der A0-Schweiz. Swiss Surg 6: 315-322

4. Helfet DL, Shonnard PY, Levine D, Borrelli J (1994) Minimally invasive plate osteosynthesis of distal fractures of the tibia.J Orthop Trauma 8: 189-194
5. Jürgens Ch, Wolter D, Queitsch C, Schultz JH (1994) Behandlungskonzepte und Ergebnisse bei nicht infizierten Pseudarthrosen an Femur und Tibia. Zentralbl Chir 119: 706-713

6. Karladani AH, Granhed H, Karrholm J, Styf J (2001) The influence of fracture etiology and type on fracture healing: a review of 104 consecutive tibial shaft fractures. Arch Orthop Trauma Surg 121: 325-328

7. Kranz HW, Wolter D, Fuchs S, Reimers N (1999) Therapie von Pseudarthrosen, Fehlstellungen und Frakturen im Unterschenkelschaftbereich mit einem Titanfixateur interne. Trauma Berufskrankh 1: 356-360

8. Megas P, Panagiotopoulos E, Skriviliotakis S, Lambiris E (2001) Intramedullary nailing in the treatment of aseptic tibial nonunion. Injury 32 : 233-239

9. Pickel H, Hofmann GO, Gonschorek O, Bühren V (2001) Umstellung posttraumatischer Fehlstellungen langer Röhrenknochen unter Verwendung von Kompressionsmarknägeln. Trauma Berufskrankh 3: 203-207
10. Seide K, Morlock MM, Schümann U, Wolter D (1999) Wirkprinzipien der winkelstabilen Platten-Schrauben-Verbindung bei Fixateurinterne-Osteosynthesen. Trauma Berufskrankh 1: 320-325

11. Templeman D, Thomas $M$, Varecka T, Kyle R (1995) Exchange reamed intramedullary nailing for delayed union and nonunion of the tibia. Clin Orthop 315: 169-175

12. Weise K, Winter E (1996) Die Rolle des Marknagels bei Pseudarthrosen und Fehlstellungen. Orthopäde 25: 247-258

13. Wolter $D$, Jürgens $C$, Wenzl M, Schümann $U$, Seide K (2001) Titanfixateur-interne-Systeme mit multidirektionaler Schraubenlage. Trauma Berufskrankh [Suppl 4] 3: S425-S428

14. Wolter D, Schümann U, Seide K (1999) Universeller Titanfixateur interne. Entwicklungsgeschichte, Prinzip, Mechanik, Implantatgestaltung und operativer Einsatz. Trauma Berufskrankh 1: 307-319 